\title{
Bayesian variable selection in modelling geographical heterogeneity in malaria transmission from sparse data: an application to Nouna Health and Demographic Surveillance System (HDSS) data, Burkina Faso
}

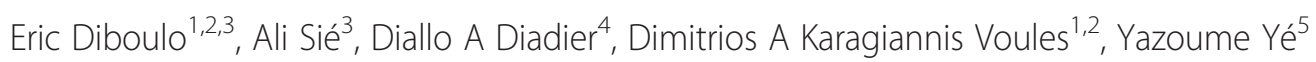
and Penelope Vounatsou ${ }^{1,2^{*}}$

\begin{abstract}
Background: Quantification of malaria heterogeneity is very challenging, partly because of the underlying characteristics of mosquitoes and also because malaria is an environmentally driven disease. Furthermore, in order to assess the spatial and seasonal variability in malaria transmission, vector data need to be collected repeatedly over time (at fixed geographical locations). Measurements collected at locations close to each other and over time tend to be correlated because of common exposures such as environmental or climatic conditions. Non- spatial statistical methods, when applied to analyze such data, may lead to biased estimates. We developed rigorous methods for analyzing sparse and spatially correlated data. We applied Bayesian variable selection to identify the most important predictors as well as the elapsing time between climate suitability and changes in entomological indices.

Methods: Bayesian geostatistical zero-inflated binomial and negative binomial models including harmonic seasonal terms, temporal trends and climatic remotely sensed proxies were applied to assess spatio-temporal variation of sporozoite rate and mosquito density in the study area. Bayesian variable selection was employed to determine the most important climatic predictors and elapsing (lag) time between climatic suitability and malaria transmission. Bayesian kriging was used to predict mosquito density and sporozoite rate at unsampled locations. These estimates were converted to covariate and season-adjusted maps of entomological inoculation rates. Models were fitted using Markov chain Monte Carlo simulation.
\end{abstract}

Results: The results show that Anophele. gambiae is the most predominant vector (79.29\%) and is more rain-dependant than its sibling Anophele. funestus (20.71\%). Variable selection suggests that the two species react differently to different climatic conditions. Prediction maps of entomological inoculation rate (EIR) depict a strong spatial and temporal heterogeneity in malaria transmission risk despite the relatively small geographical extend of the study area.

Conclusion: Malaria transmission is very heterogeneous over the study area. The EIR maps clearly depict a strong spatial and temporal heterogeneity despite the relatively small geographical extend of the study area. Model based estimates of transmission can be used to identify high transmission areas in order to prioritise interventions and support research in malaria epidemiology.

Keywords: Bayesian, Zero-inflated, Negative binomial, Stochastic search variable selection, Entomological inoculation rate, Burkina Faso, Sub-Saharan Africa, Nouna HDSS, Lag time

\footnotetext{
* Correspondence: penelope.vounatsou@unibas.ch

${ }^{1}$ Swiss Tropical and Public Health Institute, Basel, Switzerland

¿University of Basel, Basel, Switzerland

Full list of author information is available at the end of the article
} 


\section{Background}

Malaria is endemic in the majority of sub-Saharan Africa. It is transmitted from human to human via bites of mosquitoes infected with malaria parasites. A favorable environment and a complex system of malaria vectors and parasites maintain this endemicity. The mosquito development and survival strongly depend on prevailing climatic and environmental factors which in turn influence malaria transmission [1]. The species of vectors and their densities, the species of the malaria parasites, the number of infected bites a human received per night (a parameter known as the entomological inoculation rate, EIR) can change from place to place and according to the season. Therefore, malaria distribution is very heterogeneous within a geographical area and prone to between and within village variation $[2,3]$.

There are two main species of malaria vector, Anopheles gambiae and Anopheles funestus. They differ in among others things, the type of water bodies in which they lay their eggs, their propensity to bite humans, the length of time for which they survive, the place where they rest after feeding, and time of the day when they bite. An. gambiae, the most efficient malaria vector, breeds in rice fields, sunlit pools both natural and man-made, and puddles. It is mainly endophilic (rests indooors) and also endophagic (feeds indoors) and favors pools produced by rainfall. $A n$. funestus prefers shaded habitats and breeds in permanent waters, especially with vegetation. It bites humans and domestic animals and is both endophilic and exophilic [4]. Understanding the vector species' behavior and their interrelation with the environment is of prime importance in order to develop timely and effective intervention programs.

There is an elapsing time between climatic suitability, abundance of mosquito densities and onset of transmission. Changes in entomological parameters such as EIR depend on lag times and therefore it is important to take lag time into account in order to deliver timely and tailored interventions. A number of studies have used remote sensing (RS) climatic and environment proxies together to identify species-specific climatic predictors however few rigorously incorporate lag times into the analysis. RS data are often summarized by a long term average over a period of time prior to entomological data collection which is often considered as fixed rather than estimated by the data $[5,6]$.

Estimating the lag times is not only important for delivering interventions but also for obtaining good predictive models to assess the distribution of mosquitos' density. Entomological data are sparse and clustered in space and time due to spatial clustering of the environmental exposures and seasonality in transmission. Often the data include a large number of zeros (i.e. mosquito presence and/or infected) especially during the dry season.
Zeros with frequencies higher than those expected by the data distribution (for counts or proportions) often lead to overdispersion and poor fit if they are not taken into account. Zero-Inflated (ZI) models provide a flexible way to address this problem [7] by assuming that only a proportion of the zeros arise from the data distribution and the remaining ones are "structural" (i.e. they appear with probability one).

Bayesian geostatistical models have been used to take into account spatio-temporal variation and zero-inflation in entomological data $[5,6]$; however lag times in climatic factors have not been rigorously incorporated into the modelling. Furthermore, selection of the climatic predictors to be included in the model is based on standard variable selection methods, which ignore spatio-temporal correlation. Recently, Bayesian variable selection methods have been used in modeling geostatistical survey data to identify the most important predictors of disease risk [8,9], however these methods have not been applied in modelling entomological data.

In this study, we apply zero-inflated models and introduce Bayesian variable selection to determine the elapsing time between climate suitability and malaria transmission and develop predictive models of EIR taking into account spatio-temporal heterogeneity and seasonality in terms of mosquito density and infectivity (sporozoite rate). We also determine the most important climatic predictors associated with the occurrence of the most predominant malaria vector species and transmission using data from the Nouna district in Burkina Faso.

\section{Methods}

The data that motivated the present work were collected at Nouna Health and Demographic Surveillance System as part of the International Network for the Demographic Evaluation of Populations and Their Health-Malaria Transmission Intensity and Mortality Burden across Africa (INDEPTH-MTIMBA) protocol. INDEPTH-MTIMBA was a multi-centre project during 2001-2004 aimed at studying the relationship between the intensity of malaria transmission and all-cause as well as malaria specific mortality taking into account the influence of malaria control activities in each participant site. The Nouna HDSS is run by the Centre de recherche en santé de Nouna (CRSN, Nouna Health Research Center) and located in the Nouna health district's catchment area in northwest Burkina Faso, $300 \mathrm{~km}$ away from the capital city, Ouagadougou. Relative to the health district, the HDSS catchment area is located southeast.

The Nouna HDSS area is characterized by a Sub-Saharan climate with a mean annual rainfall of approximately $800 \mathrm{~mm}$ with fairly constant average daily minimum $\left(20-28.1^{\circ} \mathrm{C}\right)$ and maximum temperature $\left(29.5-37.2^{\circ} \mathrm{C}\right)$ throughout the year. Rainfall occurs from May to 
September. The entire region consists of "Plateaux" with gentle slopes and drained by several small semipermanent streams.

The HDSS area is about $1,775 \mathrm{~km}^{2}$ with the specificity of covering both rural and semi-urban areas.

The population is about 90,000 residing in 11,750 households across 58 villages and Nouna town. Subsistence farming is the predominant occupation. Malaria is holo-endemic and is known for a seasonal recrudescence during the rainy season, at which time it accounts for the main cause of fever and mortality in the district [10]. During the dry season, in February and March, lower respiratory infections are the main cause of morbidity, due to the relatively cool temperatures and strong winds, which bring up dust and dirt.

\section{Entomological data}

Entomological data were collected using the Center for Disease Control and Prevention (CDC) light traps from 10 randomly selected compounds (from the HDSS database) over two consecutive nights every two weeks during the study period (September 2001- December 2003). In each house, a light trap was hung at about $1.5 \mathrm{~m}$ above the floor next to the bed of an index person and mosquitoes were collected for two consecutive nights. The sleeping place was covered with a bed net to protect the index person from mosquito bites. Other people in the same room without bed nets were also provided with untreated nets for these specific nights. Light traps were operated from dawn to dusk. All Anopheles mosquitoes captured were identified morphologically [11], stored and dried in vials with silica gel until they could be transported to the laboratory for further testing. The head and thorax of each anopheline was tested singly for $P$. falciparum sporozoites using a standard enzymelinked immunosorbent assay (ELISA) [11]. To assess the seasonal pattern, data were summarized by location and calendar month. This implied that all surveys data collected within the same calendar month from a specific location (compound) were collapsed (mosquito density/ tested and positive) into a single observation resulting in 160 and 285 unique locations respectively for sporozoite data of An. funestus and An. gambiae and 550 unique locations for density data for both species.

\section{Environmental and climatic data}

Remote sensing data were used as proxies of climatic and environmental conditions. The predictors used, sources extracted and their spatio-temporal resolution are given in Table 1.

To account for the environment-lagged effects on changes in mosquito density and infectivity, environmental factors were extracted up to three months prior to the month of mosquito collection for each surveyed location. Based on the biological plausibility (latent periods in the mosquito and parasite life cycle), six lag variables were constructed for each environmental factor (i.e. normalized difference vegetation index (NDVI), day land surface temperature (LST), night LST and rainfall) by averaging its values over the following periods: current month of the mosquito collection $(\operatorname{Lag} 0)$, one and two month (s) prior to collection (Lag1, Lag2, respectively), average during the current and one previous month (Lag3), average during three months prior to the current one (Lag4) and average during the current and the two previous months (Lag5).

\section{Description of methods}

We followed the approach by [5] and developed zeroinflated binomial (ZIB) and zero-inflated negative binomial (ZINB) models to model sporozoite rate (proportion of infected mosquitoes) and mosquito densities, respectively. We extended the methodology by introducing Bayesian variable selection to identify the most important climatic factors related to malaria transmission and take into account lag times between climatic suitability and malaria transmission. Four models were fitted separately to An. funestus and An. gambiae data to obtain species-specific surfaces of mosquito density and sporozoite rate within the study area. The overall EIR estimate at a given location and month are based on the mean number of infected mosquitoes (from both species) multiplied by the conversion factor. Modeling details are given bellow.

Modeling sporozoite rate using zero-inflated binomial (ZIB) Let $N_{i t}, Y_{i t}^{(1)}$ be the number of tested and number of positive mosquitoes, respectively at location $i, i=1, \ldots n$

Table 1 Sources of environmental and climatic predictors

\begin{tabular}{|c|c|c|c|c|}
\hline Source & Predictor & Period & Spatial resolution & Temporal resolution \\
\hline $\begin{array}{l}\text { Moderate Resolution Imaging } \\
\text { Spectroradiometer (MODIS) Terra }\end{array}$ & Day \& Night Land Surface Temperature (LST) & $2001-2003$ & $1 \times 1 \mathrm{~km}^{2}$ & 8 days \\
\hline $\begin{array}{l}\text { Moderate Resolution Imaging } \\
\text { Spectroradiometer (MODIS) Terra }\end{array}$ & Normalized Difference Vegetation Index (NDVI) & $2001-2003$ & $0.25 \times 0.25 \mathrm{~km}^{2}$ & 16 days \\
\hline Africa Data Disseminating Services & Rainfall & $2001-2003$ & $8 \times 8 \mathrm{~km}^{2}$ & 10 days \\
\hline Health Mapper & Water Bodies (Permanent \& semi-permanent) & - & $1 \times 1 \mathrm{~km}^{2}$ & na \\
\hline
\end{tabular}


and month $t$ and $X_{i t}$ is the set of predictors. We consider that $Y_{i t}^{(1)}$ arises from a ZIB distribution, that is $Y_{i t}^{(1)} \sim Z I B$ $\left(N_{i t}, p_{i t}, \pi_{i t}^{(1)}\right)$ where $p_{i t}$ is the proportion of infected mosquitoes known as the sporozoite rate. Malaria seasonality introduces a large number of zero infected mosquitoes. The ZIB distribution assumes that a proportion $\pi_{i t}^{(1)}$ (i.e. mixing proportion) of those zeros are "structural" (not random) and the remaining ones are present in the data with the frequency defined by the binomial distribution [7]. We model the relation between the sporozoite rate $p_{i t}$ and the environmental predictors via the logistic regression equation, $\operatorname{logit}\left(p_{i t}\right)={\underset{\sim}{i t}}_{i t}^{T} \beta^{(1)}$ where $\beta_{\sim}^{(1)}$ is the set of regression coefficients. We also assume that the mixing proportion of zeros is also influenced by climatic factors $Z_{i t}^{T}$ which we introduce into the modelling by the equation $\operatorname{logit}\left(\pi_{i t}^{(1)}\right)={\underset{\sim}{i t}}_{\sim}^{T} \gamma_{\sim}^{(1)}$, where $\underset{\sim}{\gamma^{(1)}}$ is the set of corresponding coefficients.

\section{Modeling mosquito densities using zero-inflated negative binomial (ZINB)}

Let $Y_{i t}^{(2)}$ be the number of mosquitoes trapped at location $i$ and time $t$. We assume that $Y_{i t}^{(2)}$ arises from a ZINB distribution, $Y_{i t}^{(2)} \sim \operatorname{ZINB}\left(\mu_{i t}, r, \pi_{i t}^{(2)}\right)$ with $\mu_{i t}$ and $r$ corresponding to the mean mosquito count and variance of the negative binomial distribution [12]. $\pi_{i t}^{(2)}$ corresponds to the mixing proportion modelling the "excess zeros". As defined above it is considered that a proportion of the zero mosquito counts is "structural" and the remaining $1-\pi_{i t}^{(2)}$ arise from the negative binomial distribution. We model the relation between the mean mosquito density $\mu_{i t}$, mixing proportion of zeros $\pi_{i t}^{(2)}$ and climatic predictors by the equations $\log \left(\mu_{i t}\right)={\underset{\sim}{i t}}_{i}^{T} \beta_{\sim}^{(2)}$ and $\operatorname{logit}\left(\pi_{i t}^{(2)}\right)={\underset{\sim}{i t}}_{i}^{T}{\underset{\sim}{(2)}}^{(2)}$ where ${\underset{\sim}{\beta}}^{(2)}$ and ${\underset{\sim}{\gamma}}^{(2)}$ the regression coefficients.

\section{Modeling spatio-temporal heterogeneity}

We extend the above formulation to include seasonality, spatial and temporal correlation on the sporozoite rates as well as the mosquito density, that is, $\log i t\left(p_{i t}\right)={\underset{\sim}{i t}}_{\sim}^{T} \beta^{(1)}+$

$f_{(1)}(t)+\phi_{i}^{(1)}+\varepsilon_{t}^{(1)}$ and $\log \left(\mu_{i t}\right)={\underset{\sim}{X}}_{i t}^{T} \beta_{\sim}^{(2)}+f_{(2)}(t)+\phi_{i}^{(2)}+$ $\varepsilon_{t}^{(2)}$, where $f_{(k)}(t)$ captures seasonal patterns, $\phi_{i}{ }^{(k)}, \varepsilon_{t}^{(k)}$ model spatial and temporal correlation respectively. The values of the index $k$ correspond to the sporozoite models for An. funestus $(k=1)$, An. gambiae $(k=2)$ and mosquito density models for An. funestus $(k=3)$ and An. gambiae $(k=4)$ respectively. We assume a stationary Gaussian spatial process that is, spatial correlation is considered to be a function of distance only and not of the locations themselves an autoregressive process of order assuming that temporal correlation is present only between successive time points to capture temporal correlation. Seasonal trends $f_{(k)}(t)$ are modeled via a trigonometric function with a period $T=12$ months, $f_{(k)}(t)=a_{1 k} \cos \left(\frac{2 \pi}{T} t\right)+a_{2 k} \sin$ $\left(\frac{2 \pi}{T} t\right), t=1, \ldots, 12$. The peak months of the wet and dry season are calculated by $t_{k}=\arctan \left(a_{1 k} / a_{2 k}\right) \times(T / 2 \pi)$ and $\left(t_{k}+T / 2\right)$, respectively [13].

\section{Determining important predictors and lag times using variable selection}

Bayesian variable selection was carried out to determine the most important climatic factors including distance to water bodies and lag variables for each climatic factor (NDVI, day LST, night LST, Rainfall) using a variable selection approach known as stochastic search [14]. In particular, for each predictor $\underset{\sim}{X}$ we introduce a binary indicator parameter $I_{p}$ suggesting presence $\left(I_{p}=1\right)$ or absence $\left(I_{p}=0\right)$ of the predictor from the model. Furthermore, we assume a mixture prior for the corresponding regression coefficient $\beta_{p}$ that is $\beta_{p} \sim\left(1-I_{p}\right) N\left(0, v_{0} \tau_{p}^{2}\right)+I_{p}$ $N\left(0, \tau_{p}^{2}\right)$ proposing a non-informative prior for $\beta_{p}$ in case $X_{p}$ is included in the model and an informative normal prior with a variance close to zero (i.e. $v_{0}=10^{-3}$ ) shrinking $\beta_{p}$ to zero if $\underset{\sim}{X_{p}}$ is excluded from the model. A Bernoulli prior is assumed for the indicator, $I_{p} \sim B e(0.5)$. For climatic variables with lag effects we introduce a multivariate binary indicator with categories corresponding to the six lag periods and an additional category to allow exclusion of that variable. In this case we consider a multinomial prior for the indicator with equal probabilities among the categories. Variable selection was applied on the climatic factors included in both parts of the models, i.e. the mixing proportion of zeros and the mean parameters of the data distributions. The predictors that are identified as important are those with posterior inclusion probability greater than or equal to 50\% [15].

Model fit was carried out using Markov chain Monte Carlo simulation. We ran a two-chain Gibbs sampler over 100,000 iterations using a burn-in of 5000 iterations. Convergence was assessed by the Gelman and Rubin diagnostic [16] and kernel density plots using the coda routine in R.

Details on remaining prior distribution and implementation are given in the Additional file 1: Figure S1. The analysis was carried out in OpenBUGS version 3.2.3 (Imperial College and Medical Research Council, London, UK).

\section{Entomological inoculation rate (EIR)}

Bayesian kriging [17] was used to predict the mosquito density and the number of infected mosquitoes for each 


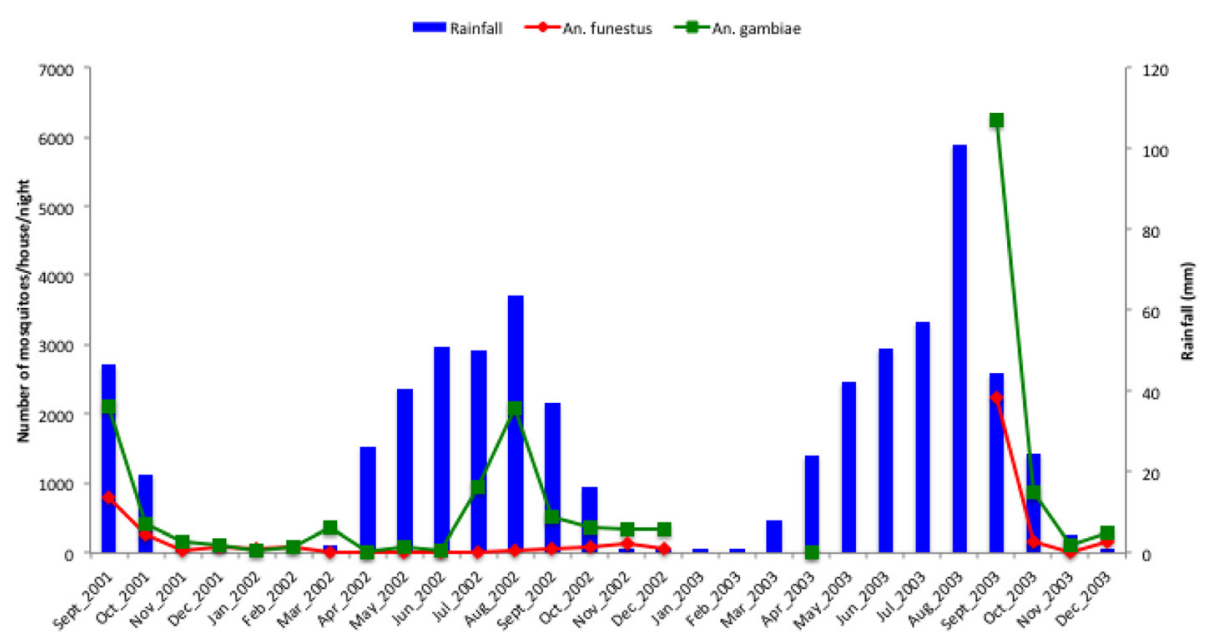

Figure 1 The distributions of the species-specific densities and rainfall throughout the study period.

species over a regular grid of 33605 pixels at $250 \times 250 \mathrm{~m}^{2}$ spatial resolution covering the study area. The entomological inoculation rate (EIR) is defined as the product of human biting rate and the sporozoite rate. The human biting rate is the mean number of bites received per host and per night. It is approximated by mosquitoes captured using human landing catches (host-seeking mosquitoes). We used a conversion factor of 1.605 [18] to transform light trap catches densities into human landing catches. The light trap density was calculated by dividing the number of mosquitoes caught using the CDC light traps by the number of trap-nights. At a specific pixel $i$ and month $t$, a sample of size 1,000 was drawn from the predicted posterior distribution of the species-specific mosquito densities using the zero-inflated negative binomial data distribution. In addition, a sample of the number of infected mosquitoes (from each species) was simulated from the predictive posterior distribution of sporozoite rates based on the zero-inflated binomial with a binomial count equal to the predicted mosquito density. The overall EIR estimate at a given location and month is based on the sample-based mean number of infected mosquitoes (from both species) multiplied by the conversion factor. Bayesian kriging was done in Fortran 95 (Digital

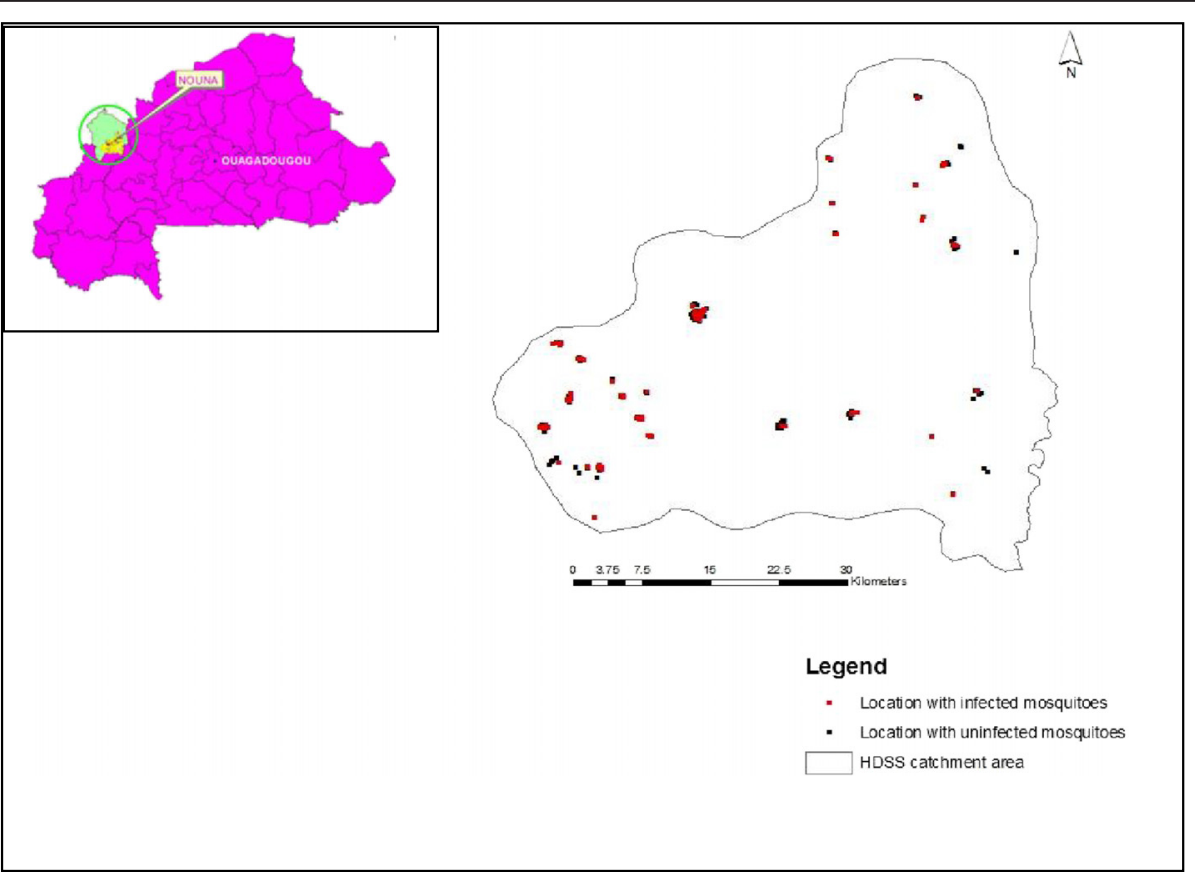

Figure 2 Geographical locations (top left) and surveyed locations with infected and uninfected mosquitoes. 


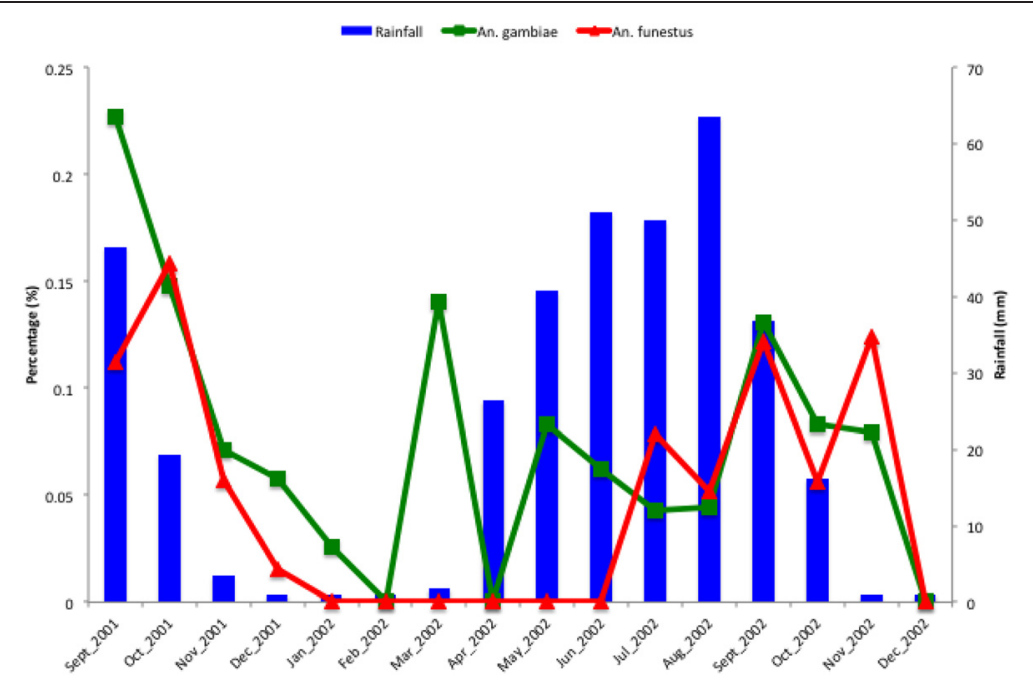

Figure 3 Monthly patterns of species-specific infectivity and rainfall.

Equipment Corporation) using standards numerical libraries (Numerical Algorithms Group Ltd.).

\section{Model validation}

Model fit was carried out on a randomly selected locations subset $(85 \%)$ of the dataset (training set). The remaining $15 \%$ was used for model validation (testing test). These subsets were selected by assigning a uniform distribution on the locations. The predictive performance of the models was assessed by calculating the proportion of test locations with the outcome variable included in the credible intervals $(\mathrm{CI})$ with varying probability coverage ranging from $5 \%$ to $95 \%$ of the posterior predictive distribution and the mean square error between the observed and predicted data [19].

\section{Results}

\section{Vectorial density}

A total of 13,132 anopheline mosquitoes were trapped in 550 unique locations over the study period. An. ${ }^{\circ}$ gambiae was the predominant vector species representing $79 \%$ of the total Anopheles mosquitoes collected. The remaining

Table 2 Lag times and predictors selected by the variable selection

\begin{tabular}{|c|c|c|c|c|}
\hline $\begin{array}{l}\text { Model } \\
\text { Binomial component }\end{array}$ & $\begin{array}{l}\text { Zero-inflated binomial } \\
\text { An. funestus }\end{array}$ & $\begin{array}{l}\text { Zero-inflated binomial } \\
\text { An. gambiae }\end{array}$ & $\begin{array}{l}\text { Zero-inflated negative binomial } \\
\text { An. funestus }\end{array}$ & $\begin{array}{l}\text { Zero-inflated negative binomial } \\
\text { An. gambiae }\end{array}$ \\
\hline \multicolumn{5}{|l|}{ Parameter } \\
\hline Rainfall & Lag 3 & Lag 0 & Lag 0 & Lag 5 \\
\hline Vegetation (NDVI) & - & - & Lag 0 & - \\
\hline Day temp (LSTD) & $\operatorname{Lag} 4$ & Lag 3 & Lag 1 & - \\
\hline Night temp (LSTN) & - & Lag 5 & - & Lag 4 \\
\hline Distance to water body & Yes & No & No & No \\
\hline \multicolumn{5}{|l|}{ Mixing Proportion } \\
\hline Rainfall & - & - & - & - \\
\hline Vegetation (NDVI) & - & - & - & - \\
\hline Day temp (LSTD) & - & - & Lag 1 & $\operatorname{Lag} 2$ \\
\hline Night temp (LSTN) & - & - & Lag 1 & Lag 5 \\
\hline Distance to water body & Yes & Yes & Yes & No \\
\hline
\end{tabular}

\section{Legend:}

Lag 0: Average over the current month.

Lag 1: Average of the environmental covariate over the previous month.

Lag 2: Average of the environmental covariate over second previous month.

Lag 3: Average of the environmental covariate over the current and the previous month.

Lag 4: Average of the environmental covariate over the previous and the second previous month.

Lag 5: Average of the environmental covariate over the current, previous and the second previous month. 
Table 3 Posterior estimates obtained from the geostatistical zero-inflated negative binomial (ZINB) models

\begin{tabular}{|c|c|c|}
\hline Parameters & $\begin{array}{l}\text { An. funestus } \\
\text { Median }(95 \% \mathrm{BCl})\end{array}$ & $\begin{array}{l}\text { An. gambiae } \\
\text { Median }(95 \% \mathrm{BCl})\end{array}$ \\
\hline Intercept & $-0.38(-1.09,0.46)$ & $1.85(-0.11,3.56)$ \\
\hline Year2 & $0.24(-0.64,1.07)$ & $-1.01(-2.75,0.39)$ \\
\hline Rainfall & $0.87(0.021,1.71)$ & $-2.33(-4.67,-0.2)$ \\
\hline Vegetation (NDVI) & $1.12(0.63,1.65)$ & - \\
\hline Day temp (LSTD) & $-0.78(-1.56,0.00)$ & - \\
\hline Night temp (LSTN) & - & $-1.3(-1.9,-0.64)$ \\
\hline Amplitude & $3.53(3.50,3.56)$ & $5.88(5.83,5.93)$ \\
\hline Shift/phase & $0.648(0.645,0.653)$ & $-1.159(-1.163,-1.155)$ \\
\hline Dispersion ( $r$ ) & $0.45(0.32,0.63)$ & $0.93(0.71,1.24)$ \\
\hline Spatial variation & $0.90(0.33,2.03)$ & $0.54(0.22,1.13)$ \\
\hline Range $(\mathrm{km})^{\mathrm{a}}$ & $1(3,83)$ & $5(1,35)$ \\
\hline Temporal variation & - & $0.76(0.48,1.26)$ \\
\hline \multirow[t]{3}{*}{ Parameters } & \multicolumn{2}{|l|}{ Mixing proportion } \\
\hline & An. funestus & An. gambiae \\
\hline & Median (95\% BCI) & Median (95\% BCI) \\
\hline Intercept & $-14.42(-27.08,-3.57)$ & $-11.06(-18.80,-3.47)$ \\
\hline Year2 & $0.24(-0.64,1.07)$ & $4.42(-5.67,11.66)$ \\
\hline Distance to water body & $-3.52(-12.43,3.51)$ & - \\
\hline Rainfall & - & - \\
\hline Vegetation (NDVI) & - & - \\
\hline Day temp (LSTD) & $4.14(-9.38,21.23)$ & $3.21(0.89,5.89)$ \\
\hline Night temp (LSTN) & $5.13(-5.08,14.83)$ & $-1.99(-5.57,-0.57)$ \\
\hline Amplitude & $21.92(21.69,22.16)$ & $4.03(3.96,4.18)$ \\
\hline Shift/phase & $1.06(1.05,1.07)$ & $2.25(2.22,2.27)$ \\
\hline
\end{tabular}

${ }^{a}$ minimum distance in kilometer at which the spatial correlation remains important, $\mathrm{BCl}=$ Bayesian Credible Interval.

$21 \%$ consisted of An. funestus. About 35\% of the survey locations had no An. gambiae mosquitoes. This percentage reaches $41 \%$ for $A n$. funestus. The peak collecting period for An. gambiae species coincided with the peak of the rainy season (August) while for An. funestus it was in September. However the density of An. funestus remained comparatively low throughout the study period. The distributions of the species-specific densities and rainfall throughout the study period are given in Figure 1.

\section{Mosquito infectivity}

A total of 5,668 mosquitoes were tested for the presence of circumsporozoite antigens out of which 4,230 (74.64\%) were An. gambiae species. The overall sporozoite rate (SR) was $7.64 \%$ (95\% CI 7.63-7.65). Plasmodium falciparum infection was detected in $9.24 \%$ of An. gambiae species and $2.92 \%$ of An. funestus species. Sporozoite rates tend to be higher in the Western part of the study area. Figure 2 presents the geographical location of the study area (top left) and surveyed locations with infected and uninfected mosquitoes. The monthly patterns of infected mosquitos and rainfall by species are depicted in Figure 3.

\section{Variable selection}

Results of the variable selection in Table 2 indicate that rainfall and vegetation during the current month (Lag0), and day temperature during the month preceding the collection (Lag1) are important predictors of An. funestus density. The rise of An. gambiae density depends on suitable climatic conditions over a longer period of time such as rainfall during the current and previous two months and night temperatures during the previous two months. The proportion of "structural" or "excess" zeros is influenced by day and night temperatures for both species. However, for An. gambiae densities, the mixing proportion is associated with a longer lag time, that is second month (Lag2) and two previous and current months (Lag5) for day and night temperatures, respectively. Furthermore, distance to water bodies appears to be an important predictor of the mixing proportion of zeros for An. funestus densities.

The most important climatic predictors of the sporozoite rates of An. funestus are rainfall during the current and previous month (Lag3) and day temperature during the two previous months (Lag4). Rainfall of the month of collection ( $\operatorname{Lag} 0)$ and more distant lag times for day (Lag3 corresponding to the current and previous month) and night temperatures (Lag5, i.e. current and the two previous months) appear to be the most important predictors of An. gambiae sporozoite rates. Distance to water body is the only important predictor of the proportion of "excess" zeros in the sporozoite rates for both species.

\section{Model-based vectorial density}

Positive effects of rainfall and vegetation during the current month showed important associations with the density of An. funestus. The distance at which the spatial correlation is less than $5 \%$ is equal to $10 \mathrm{~km}$ (95\% Bayesian credible interval $(\mathrm{BCI}): 3-83 \mathrm{~km})$. The phase of 0.65 radials suggested that the peak of the An. funestus density occurs in the month of September and the minimum in the month of March. The effect of predictors associated to the mixing proportion of the zero-inflated distribution was not important. The probability of the excess zeros is highest in the month of November and the lowest in the month of April.

Rainfall (during the current and two previous months) and night temperature (during the two previous months) are important predictors, negatively associated with $A n$. gambiae density. Spatial correlation is not important $(<0.05)$ beyond $5 \mathrm{~km}(95 \%$ BCI: $1-35 \mathrm{~km})$. The temporal and the spatial variations are respectively 0.76 (95\% BCI: 


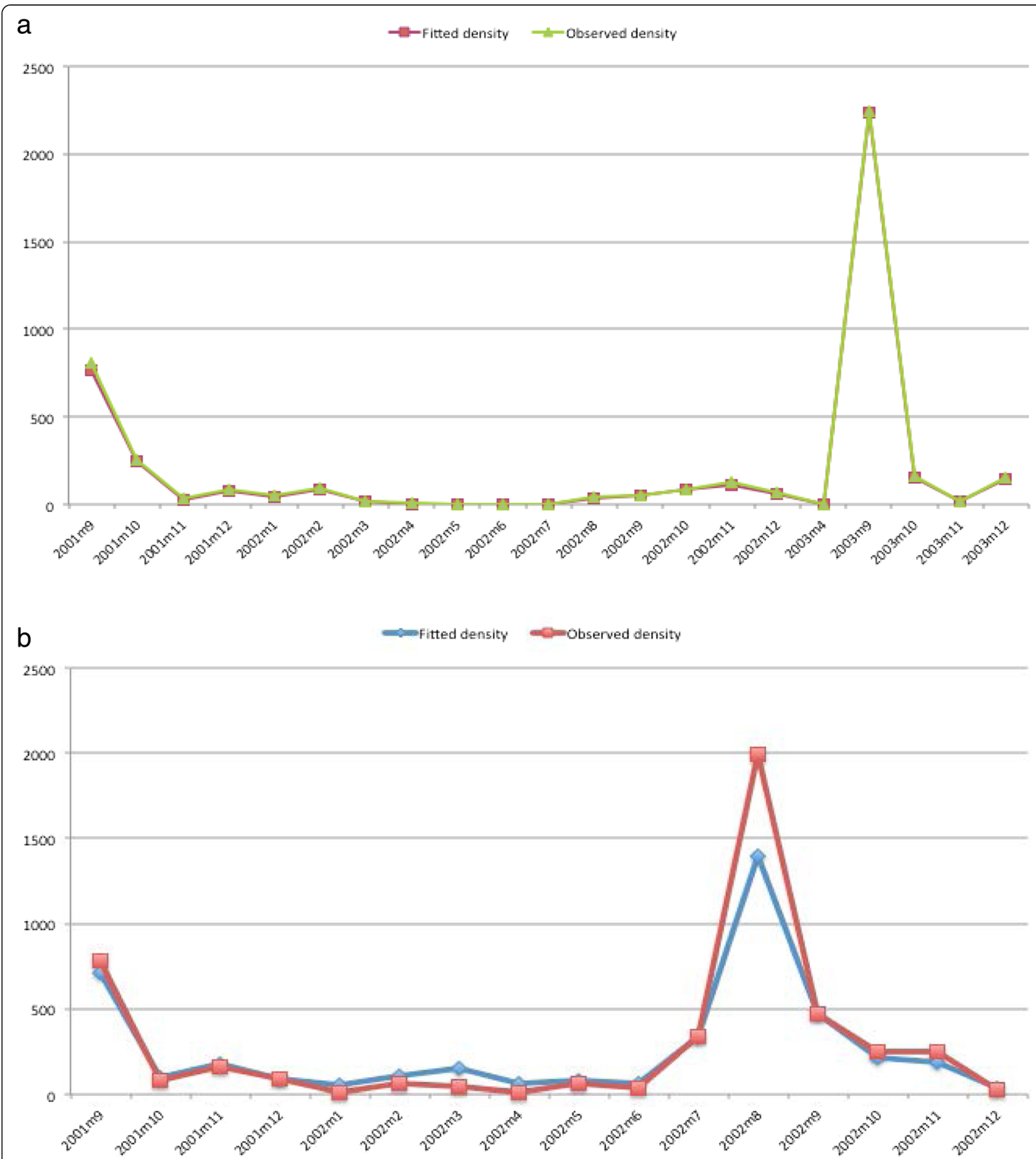

Figure 4 Monthly patterns of observed and fitted indoor residual densities. (a) Averaged over spatial locations of An. funestus. (b) Averaged over spatial locations of An. gambiae.

$0.48-1.26)$ and 0.54 (95\% BCI: 0.22-1.13). The maximum An. gambiae density occurs in the month of August and the minimum in the month of January. The probability of excess is related positively with the day temperature during the two previous months and negatively with the night temperature during the current and two previous months. The maximum probability of excess zero occurs in November and the minimum in April. Table 3 presents the posterior estimates of the ZINB model for both species. Figure 4 shows the monthly pattern of observed and fitted density (averaged over spatial locations) respectively for An. funestus and An. gambiae. 
Table 4 Posterior estimates obtained from the geostatistical zero-inflated binomial (ZIB) models

\begin{tabular}{|c|c|c|}
\hline Parameters & $\begin{array}{l}\text { An. funestus } \\
\text { Median (95\% Cl) }\end{array}$ & $\begin{array}{l}\text { An. gambiae } \\
\text { Median }(95 \% \mathrm{Cl})\end{array}$ \\
\hline Intercept & $-6.65(-14.8,-1.42)$ & $-1.82(-4.21,4.14)$ \\
\hline Year2 & $-0.67(-3.36,2.10)$ & $-0.92(-4.32,1.49)$ \\
\hline Distance to water body & $0.10(-1.54,1.83)$ & - \\
\hline Rainfall & $-1.42(-4.84,1.51)$ & $-0.16(-2.06,1.94)$ \\
\hline Vegetation (NDVI) & - & - \\
\hline Day temp (LSTD) & $-0.25(-3.53,2.64)$ & $-0.47(-1.13,0.08)$ \\
\hline Night temp (LSTN) & - & $0.05(-0.72,0.81)$ \\
\hline Amplitude & $7.25(7.12,7.38)$ & $2.50(2.45,2.54)$ \\
\hline Shift/phase & $-1.89(-1.94,-1.85)$ & $2.72(2.68,2.75)$ \\
\hline Spatial variation & $0.67(0.22,2.78)$ & $0.51(0.22,1.19)$ \\
\hline Range $(\mathrm{km})^{\mathrm{a}}$ & $0.05(0.01,0.44)$ & $0.05(0.01,0.27)$ \\
\hline Temporal variation & - & $0.99(0.53,2.26)$ \\
\hline \multirow[t]{3}{*}{ Parameters } & \multicolumn{2}{|l|}{ Mixing proportion } \\
\hline & An. funestus & An. gambiae \\
\hline & Median $(95 \% \mathrm{Cl})$ & Median $(95 \% \mathrm{Cl})$ \\
\hline Intercept & $-2.96(-20.12,11.79)$ & $-11.45(-26.0,-0.0015)$ \\
\hline Year2 & $1.88(-17.24,15.2)$ & $-3.25(-18.15,9.07)$ \\
\hline Distance to water body & $11.5(-7.67,27.29)$ & $3.546(-9.625,11.39)$ \\
\hline Rainfall & - & - \\
\hline Vegetation (NDVI) & - & - \\
\hline Day temp (LSTD) & - & - \\
\hline Night temp (LSTN) & - & - \\
\hline Amplitude & $11.09(10.88,11.30)$ & $4.03(3.96,4.10)$ \\
\hline Shift/phase & $1.19(1.15,1.23)$ & $2.25(2.22,2.27)$ \\
\hline
\end{tabular}

${ }^{a}$ minimum distance in kilometer at which the spatial correlation is significant at $5 \%, \mathrm{Cl}=$ credible interval.

\section{Model-based mosquito infectivity}

Table 4 presents the posterior estimates of the ZIB model for both species. The effects of predictors appeared not to be important for both species. The maximum of $A n$. funestus infectivity occurs in the month of August and the minimum in April. Estimates of the seasonality parameters in the mixing proportion indicate that the probability of "structural" zeros is maximum in October and minimum in the month of April.

Sporozoite rates in $A n$. gambiae take the largest and lowest values in November and April, respectively.

The probability of "structural" zeros is higher in December and lower in June. Variation in time is larger than the one in space. Furthermore, the distance at which the correlation coefficient falls below 5\% (spatial correlation) is deemed unimportant for both An. funestus and An. gambiae (95\% BCI: $1-44 \mathrm{~km}$ and 1-27 km, respectively). Figure $5 \mathrm{a}-\mathrm{b}$ shows the monthly pattern of observed and fitted sporozoite rate (averaged over spatial locations) respectively for $A n$. funestus and An. gambiae.

\section{Entomological inoculation rate (EIR)}

The annual EIR averaged across the area was 131.4 infective bites per person for 2002. Figure 6 depicts monthly EIR estimates of the median predictive posterior distribution at 250 by $250 \mathrm{~m}^{2}$ resolutions within the HDSS site. The high transmission season is during May-October; however there are some "high-transmission" areas in the western part during November. In fact, the western region has the highest EIR estimates across the whole HDSS catchment area.

\section{Model validation}

Model validation showed that $92 \%$ and $73 \%$ of the test locations had sporozoite rate falling within the 95\% credible interval estimated from the zero inflated spatial binomial model and zero inflated spatio-temporal model respectively for $A n$. funestus and An. gambiae.

Density models validation showed that $73 \%$ and $58 \%$ of the test locations had mosquito densities falling within the 95\% credible interval estimated from the zero inflated spatial negative binomial model and zero inflated spatio-temporal negative binomial model respectively for $A n$. funestus and An. gambiae density models. However the zero inflated spatio-temporal models included higher proportion of test locations in the lowest credible intervals compared to the zero inflated spatial negative binomial. Figure 7a-b shows the proportions of test locations with respectively sporozoite rate and mosquito density falling in between $5 \%$ and $95 \%$ credible intervals of the posterior predictive distribution.

\section{Discussion}

In this study we described and quantified malaria transmission heterogeneity in the Nouna HDSS, using a comprehensive entomological dataset and rigorous spatiotemporal models, which include Bayesian variable selection and take into account zero-inflation. The models determine the elapsing time between climate suitability and malaria transmission and estimate spatio-temporal patterns of transmission. Malaria transmission is mainly driven by two efficient vectors namely Anopheles gambiae and $A n$. funestus, which co-exist geographically across the study area. The transmission fluctuated over the study period indicating seasonal, spatial and temporal variation within such a small geographic extend $\left(1775 \mathrm{~km}^{2}\right)$. These findings corroborate previous studies concluding a very heterogeneous malaria distribution which is prone to great variations between villages and compounds $[2,3,6]$. Transmission intensity measured by EIR in Nouna HDSS was high $(>100 \mathrm{ib} / \mathrm{p} / \mathrm{y})$ especially in the rainy season. A 


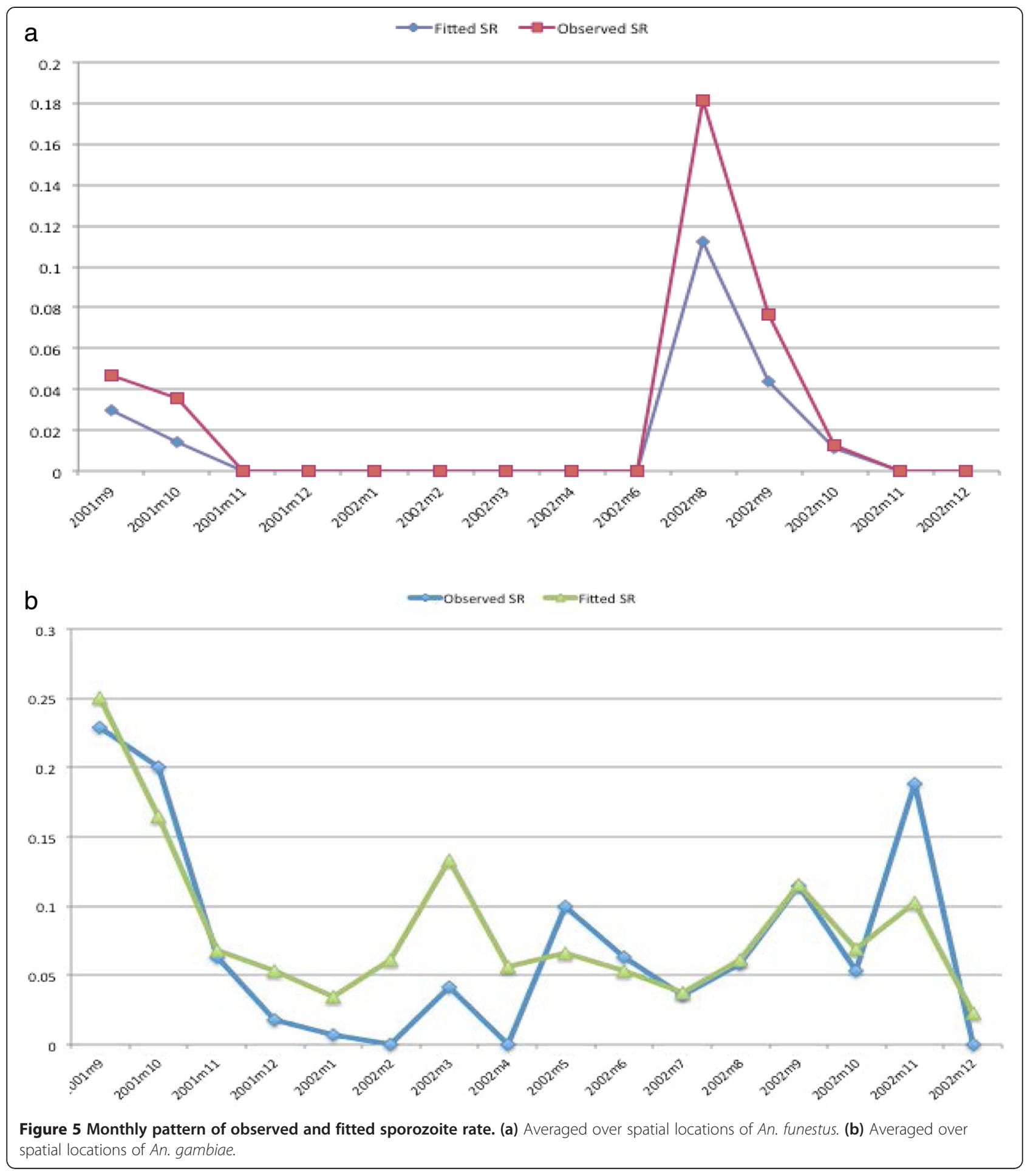

seasonal pattern was observed in mosquito densities and in sporozoite rates for both species. The high transmission season starts from May throughout October with the peak transmission occurring in September.

The negative relationship between night temperatures and $A n$. gambiae mosquito density in our results possibly imply that although the high temperatures of the study area (average daily minimum: $20-28.1^{\circ} \mathrm{C}$, maximum: $29.5-37.2^{\circ} \mathrm{C}$ ) are suitable for stable malaria transmission [20], a spell of relief from the heat mainly in the night is also a key determinant for mosquito development and survival.

Rainfall is associated with the densities of both species; however the direction of the effect is different. A 


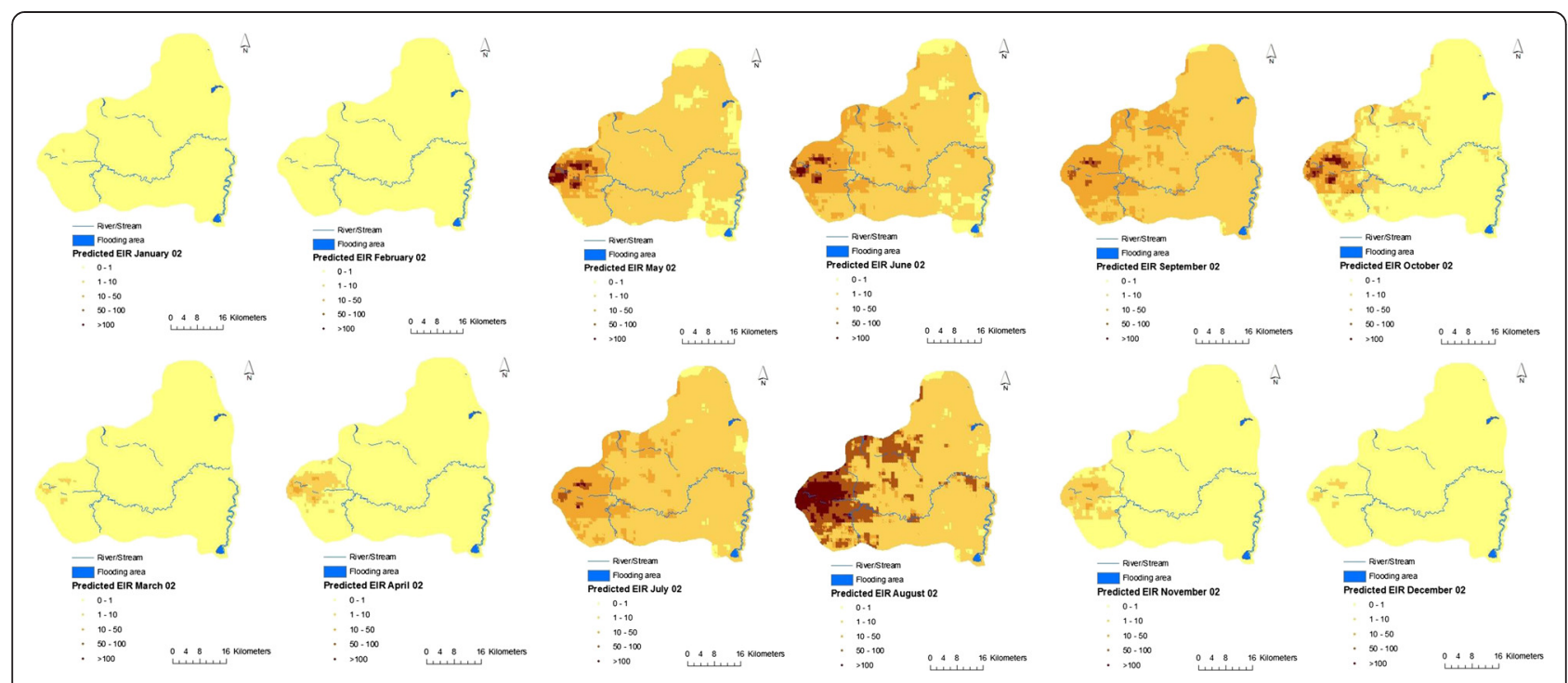

Figure 6 Monthly EIR estimates of the median predictive posterior distribution at 250 by $250 \mathrm{~m}^{2}$ resolutions.

negative association with An. gambiae density may suggest that although rainfall remains an important factor for the development of this species, consecutive heavy rainfall (over the current and the two previous months as shown by the Lag5 effect) may flush away all suitable An. gambiae breeding sites, therefore An. gambiae is a rainy-dependant species which favours temporary and shallow breeding sites. A positive association with $A n$. funestus density indicates that rainfall is important for the development and survival of this species which predominantly develops in permanent water bodies with emerging vegetation [21]. This result is consistent with the positive important association between NDVI (a proxy measure of vegetation) and An. funestus density and the lack of association between NDVI with An. gambiae density (NDVI was not identified as a potential predictor of An. gambiae density).

The shortest distance at which the spatial correlation was below $5 \%$ for sporozoite rate was $5 \mathrm{~km}$ for both species. However, for the density it is twice as much for $A n$. funestus than that for An. Gambiae in spite of wide credible intervals associated with both estimates. The negative association between rainfall and sporozoite rate (although not significant) for both species can be explained by the sporogony cycle in relation to mosquito survival. Furthermore, only adult mosquitoes that have successfully taken a blood meal carry sporozoites while many young newly emerged mosquitoes shortly after the onset of the rainy season may reduce the proportion of older ones in the population. Similar results were also found by [22] and [23].

The lack of association between distance to water bodies especially An. gambiae may be explained by the fact the water bodies considered are mostly large permanent and semi-permanent ones and does not include small breeding sites which are favoured by An. gambiae. The zero-inflated (ZI) model formulations that were adopted in our study allow us to account for the structural zeros that may arise due to some factors that have not been considered in the study. For example, vector control interventions targeting adult mosquitoes that are likely to be infective or proximity to temporary water bodies where it is likely to find many young newly emerged (not yet infective) mosquitoes.

Interestingly, high EIR estimates are observed in the western part of the study area, which is located to a large extent in shallows that are extensively used by local populations for rice cultivation. The transmission in this area remained high even during the dry season. Figure 3 in the Additional file 1: Figure S1 shows the monthly patterns of observed and fitted of sporozoite rate $(a, b)$ (c,d) and densities (e,f)-(g,h) averaged over spatial locations respectively in high and low EIR regions of the study area and for An. funestus and An. gambiae. The observed and fitted sporozoite rate and densities plots display similar patterns in both the low and high EIR regions.

The EIR maps clearly depict a strong spatial and temporal heterogeneity despite the relatively small geographical extent of the study area.

These maps are valuable tools in identifying malaria "high-transmission" areas and in prioritizing timely, control interventions. The high spatial resolution EIR estimates are also important in addressing research questions such as the relationship between malaria transmission intensity and mortality.

The lag time analysis indicated short elapsing periods between climatic suitability and rise of An. funestus densities as opposed to longer times required by An. gambiae. 

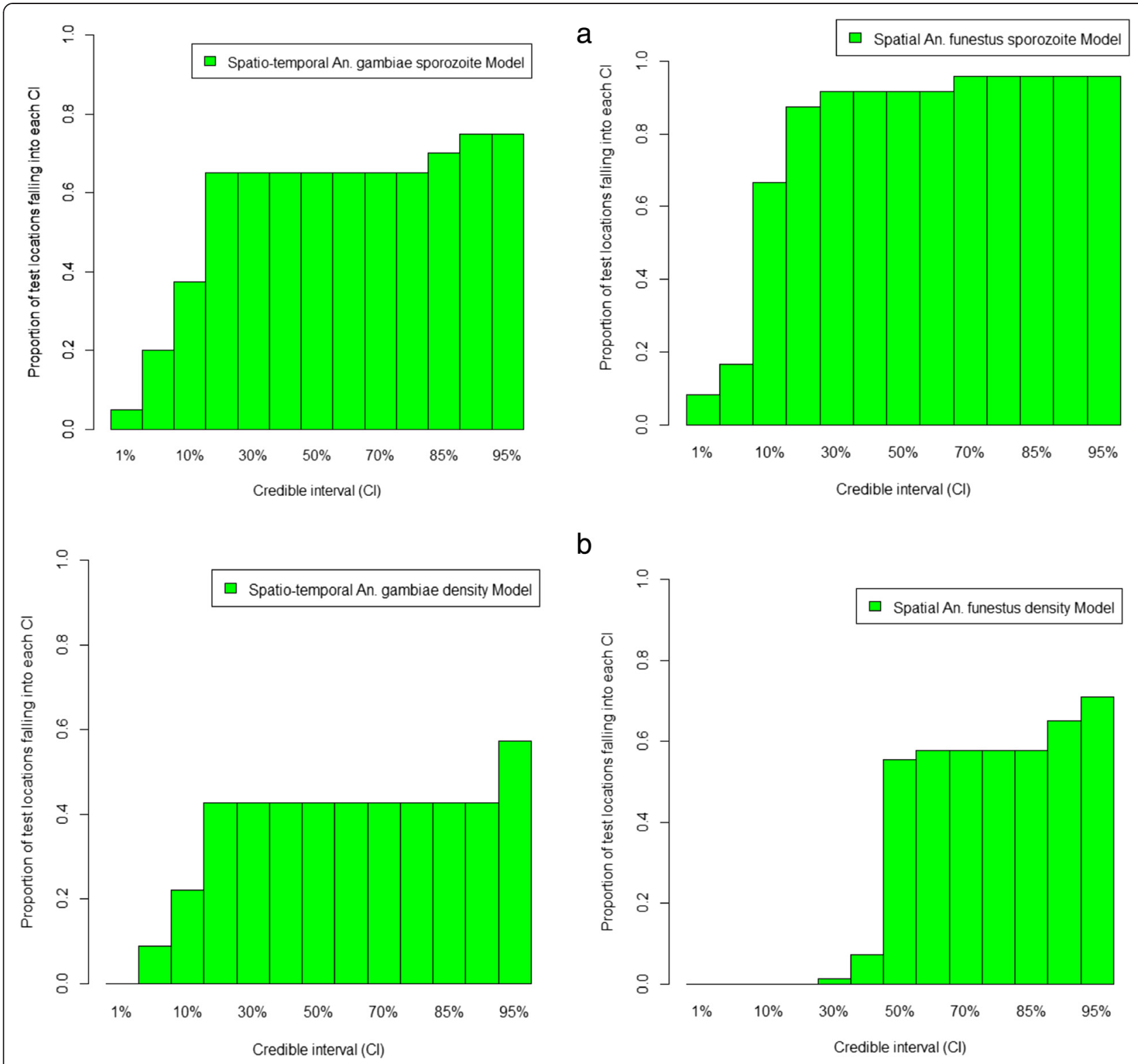

b

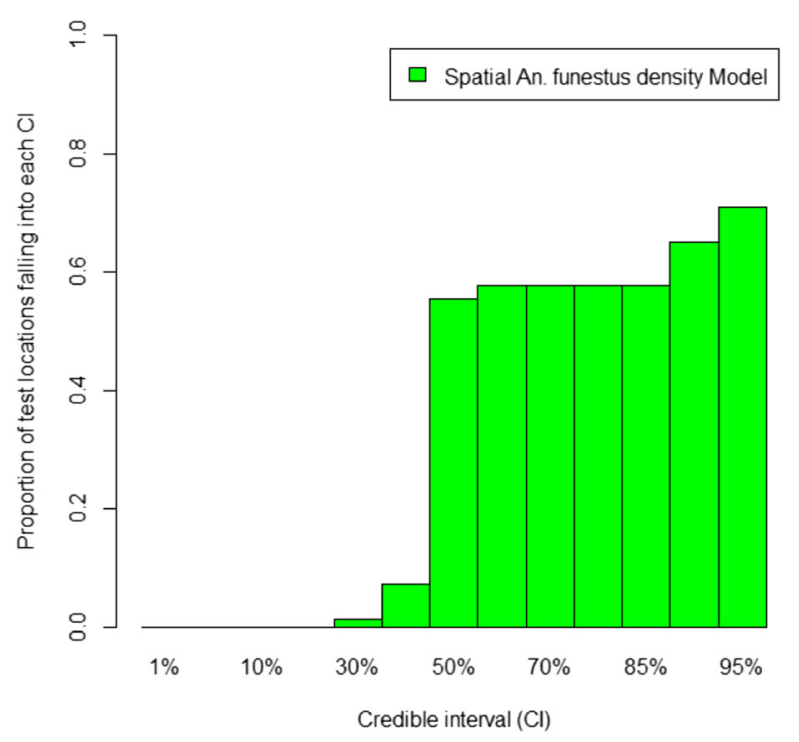

Figure 7 Proportions of test locations falling in between $\mathbf{5 \%}$ and $\mathbf{9 5 \%}$ credible intervals. (a) Proportions of test locations with sporozoite rate falling in between $5 \%$ and $95 \%$ credible intervals. (b) Proportions of test locations with mosquito density falling in between 5\% and $95 \%$ credible intervals.

A possible explanation could be that rainfall quickly dries out or it streams into shallows (in case of heavy rainfall) where water is collected for an extended period thus favouring the development of An. funestus. Suitable breeding sites for An. gambiae appear only after successive rainfalls that lead to soil saturation. Understanding the lag times between climate suitability and change in malaria transmission is important not only for delivering interventions at the right time but also for developing predictive models to support early warning systems (EWS). In many studies the choice of environmental predictors is based on biological plausibility rather than assessing whether plausibility is supported by the data generated by the study site. However, local conditions influence transmission patterns, therefore rigorous modelling approaches that take into account and estimate lag times in climatic factors are needed to increase model predictive ability.

In this study we used and systematically examined different lag structures through Bayesian variable selection implemented within a geostatistical model. Modeling lag effects via distributed lag models [24-26] is an alternative approach to the one used in this paper, however this approach assumes that the different climatic proxies are available on a daily scale or aggregated over a common 
temporal resolution (e.g. month). We are currently comparing both approaches.

To our knowledge, this is the first effort in estimating and comparing the lag time between climatic suitability and malaria transmission between the two vector species. The results improve our understanding of the dynamics of malaria transmission. However it is worth noticing that the associations found in this study area may not necessarily apply in different eco-climatic zones and further work in the area could clarify how lag effects depend on ecological zone. Model based estimates of transmission can identify high transmission areas in order to prioritise interventions and support research in malaria epidemiology.

\section{Conclusions}

This study identified the most important environmental/ climatic variables associated with malaria transmission in the Nouna region in Burkina Faso and determined the lag times between climate suitability and change in entomological indices. The prediction maps of entomological inoculation rate (EIR) surfaces depict a strong spatial and temporal heterogeneity. Our results contribute to a better understanding of the interplay between environmental/climatic conditions and malaria transmission, which is important not only for delivering interventions at the right time but also for developing predictive models to support early warning systems (EWS).

\section{Appendix}

\section{Prior distributions and model implementation}

We assume that $\phi_{i}^{(k)}$ s latent observations from a stationary Gaussian spatial process $N\left(0, \sigma_{1 k}^{2} R^{(k)}\right) \cdot R^{(k)}$ models spatial correlation as an exponential function of distance $d_{i j}$ between any pairs of locations $i$ and $j$ that is $R_{i j}^{(k)}=\exp \left(d_{i j} ; \rho_{k}\right)$ and $\rho_{k}$ is a measure of the rate of the correlation decay with distance. The value $3 / \rho_{k}$ estimates the maximum distance at which the spatial correlation is significant at 5\% [27]. The $\sigma_{1 k}^{2}$ measures the within-location variation. Temporal correlation was introduced by monthly random effects and modelled by autoregressive (AR) process of order 1 that is $\varepsilon_{t}^{(k)} \sim N\left(\theta^{(k)} \varepsilon_{t-1}^{(k)}, \sigma_{2 k}^{2}\right)$ and $\varepsilon_{t}^{(k)} \sim N\left(0, \sigma_{2 k}^{2} / 1-\theta_{k}\right) . \sigma_{2 k}^{2}$ and $\theta_{k}$ are the temporal variance and autocorrelation parameters respectively.

For the regression coefficients we adopt a noninformative normal prior distribution with large variance. We further assumed a normal prior distributions with mean zero and large variance for the coefficients of the seasonal trends, that is $a_{1 k}$ and $a_{2 k} \sim N\left(0,10^{2}\right)$. For the spatial parameters $\sigma_{k}^{2}$ and $\rho_{k}$ we adopt inverse gamma and gamma prior distributions respectively, that is $\sigma_{k}^{2} \sim I G(2.01,1.01)$ and $\rho_{k} \sim G(0.1,0.1)$. Covariates were standardized in order to acquire better correlation properties and reduce the Markov chain Monte Carlo simulation computational time [28].

Convergence was assessed by Gelman and Rubin diagnostic [16] and kernel density plots.

Exploratory analysis was carried out in STATA 11 (Stata Corporation, College Station, Texas, USA).

\section{Additional file}

\begin{abstract}
Additional file 1: Figure S1. (a): Monthly pattern of observed and fitted sporozoite rate of An. funestus: averaged over spatial locations in western (high EIR) region of the study area. (b): Monthly pattern of observed and fitted sporozoite rate of An. funestus: averaged over spatial locations in eastern (low EIR) region of the study area. (c): Monthly pattern of observed and fitted densities of An. gambiae: averaged over spatial locations in western (high EIR) region of the study area. (d): Monthly pattern of observed and fitted densities of An. gambiae: averaged over spatial locations in western (low EIR) region of the study area. (e): Monthly pattern of observed and fitted densities of An. funestus: averaged over spatial locations in western (high EIR) region of the study area. (f): Monthly pattern of observed and fitted densities of An. funestus: averaged over spatial locations in western (low EIR) region of the study area. (g): Monthly pattern of observed and fitted densities of An. gambiae: averaged over spatial locations in western (high EIR) region of the study area. (h): Monthly pattern of observed and fitted densities of An. gambiae: averaged over spatial locations in western (low EIR) region of the study area.
\end{abstract}

\section{Competing interest}

The authors declare that they have no competing interests.

\section{Authors' contributions}

PV conceived and designed the study. ED analyzed the data and drafted the manuscript. All authors gave intellectual content and critically reviewed the drafts and approved the final manuscript.

\section{Acknowledgments}

The authors are grateful to the CRSN and the principal investigator of the INDEPTH-MTIMBA project in Burkina Faso for providing the data. This work was funded by the Swiss National foundation (SNSF) Swiss Programme for Research on Global Issues for Development (R4D) project no. IZ01Z0-147286. DAKV was funded by SNSF project no. PDFMP3-137156.

\section{Author details}

${ }^{1}$ Swiss Tropical and Public Health Institute, Basel, Switzerland. ${ }^{2}$ University of Basel, Basel, Switzerland. ${ }^{3}$ Centre de Recherche en Santé de Nouna, Nouna, Burkina Faso. ${ }^{4}$ PATH Malaria Vaccine Initiatives, Dakar, Senegal. ${ }^{5}$ ICF International, Rockville, MD 20850, USA.

Received: 2 October 2014 Accepted: 21 January 2015 Published online: 22 February 2015

\section{References}

1. Yé Y. Environmental Factors and Malaria Transmission Risk: Modelling the Risk in a Holoendemic Area of Burkina Faso. Surrey, UK: Ashgate Publishing, Ltd; 2008.

2. Greenwood BM. The microepidemiology of malaria and its importance to malaria control. Trans R Soc Trop Med Hyg. 1989;83(Suppl):25-9.

3. Carter R, Mendis KN, Roberts D. Spatial targeting of interventions against malaria. Bull World Health Organ. 2000;78:1401-11.

4. Guelbeogo W, Sagnon N, Grushko O, Yameogo M, Boccolini D, Besansky N, et al. Seasonal distribution of Anopheles funestus chromosomal forms from Burkina Faso. Malar J. 2009:8:239.

5. Rumisha SF, Smith T, Abdulla S, Masanja H, Vounatsou P. Modelling heterogeneity in malaria transmission using large sparse spatio-temporal entomological data. Glob Health Action. 2014. 
6. Amek N, Bayoh N, Hamel M, Lindblade KA, Gimnig JE, Odhiambo F, et al. Spatial and temporal dynamics of malaria transmission in rural Western Kenya. Parasit Vectors. 2012;5:86.

7. Hall DB. Zero-inflated Poisson and binomial regression with random effects: a case study. Biometrics. 2000;56:1030-9.

8. Giardina F, Gosoniu L, Konate L, Diouf MB, Perry R, Gaye O, et al.. Estimating the burden of malaria in Senegal: Bayesian zero-inflated binomial geostatistical modeling of the MIS 2008 Data. PLoS ONE. 2012.

9. Chammartin F, Scholte RG, Guimarães LH, Tanner M, Utzinger J, Vounatsou P. Soil-transmitted helminth infection in South America: a systematic review and geostatistical meta-analysis. Lancet Infect Dis. 2013;13:507-18.

10. Müller O, De Allegri M, Becher H, Tiendrebogo J, Beiersmann C, Ye M, et al. Distribution systems of insecticide-treated bed nets for malaria control in Rural Burkina Faso: cluster-randomized controlled trial. PLOS ONE. 2008:3:e3182.

11. Wirtz RA, Zavala F, Charoenvit Y, Campbell GH, Burkot TR, Schneider I, et al. Comparative testing of monoclonal antibodies against Plasmodium falciparum sporozoites for ELISA development. Bull World Health Organ. 1987;65:39-45.

12. Vounatsou P, Raso G, Tanner M, N'goran EK, Utzinger J. Bayesian geostatistical modelling for mapping schistosomiasis transmission. Parasitology. 2009;136:1695-705.

13. Stolwijk AM, Straatman H, Zielhuis GA. Studying seasonality by using sine and cosine functions in regression analysis. J Epidemiol Community Health. 1999;53:235-8

14. George El, McCulloch RE. Variable selection via gibbs sampling. J Am Stat Assoc. 1993;88:881-9.

15. Barbieri MM, Berger JO. Optimal predictive model selection. Ann Stat. 2004:32:870-97.

16. Brooks SP, Roberts GO. Convergence assessment techniques for Markov chain Monte Carlo. Stat Comput. 1998:8:319-35.

17. Diggle PJ, Tawn JA, Moyeed RA. Model-based geostatistics. J R Stat Soc Ser C Appl Stat. 1998:47:299-350.

18. Lines JD, Curtis CF, Wilkes TJ, Njunwa KJ. Monitoring human-biting mosquitoes (Diptera: Culicidae) in Tanzania with light-traps hung beside mosquito nets. Bull Entomol Res. 1991;81:77-84.

19. Gosoniu L, Vounatsou P, Sogoba N, Smith T. Bayesian modelling of geostatistical malaria risk data. Geospatial Health. 2006;1:127-39.

20. Craig MH, Snow RW, le Sueur D. A climate-based distribution model of malaria transmission in sub-Saharan Africa. Parasitol Today Pers Ed. 1999;15:105-11.

21. Dia I, Wamdaogo M, Ayal D. Advances and Perspectives in the Study of the Malaria Mosquito Anopheles funestus. In: Manguin S, editor. Anopheles mosquitoes - New insights into malaria vectors. Rijeka, Croatia: InTech; 2013.

22. Amek N, Bayoh N, Hamel M, Lindblade KA, Gimnig J, Laserson KF, et al. Spatio-temporal modeling of sparse geostatistical malaria sporozoite rate data using a zero inflated binomial model. Spat Spatio-Temporal Epidemiol. 2011:2:283-90.

23. Kasasa S, Asoala V, Gosoniu L, Anto F, Adjuik M, Tindana C, et al. Spatiotemporal malaria transmission patterns in Navrongo demographic surveillance site, northern Ghana. Malar J. 2013;12:63.

24. Almon S. The distributed lag between capital appropriations and expenditures. Econometrica. 1965;33:178-96.

25. Gasparrini A, Armstrong B, Kenward MG. Distributed lag non-linear models. Stat Med. 2010:29:2224-34.

26. Gasparrini A. Distributed lag linear and non-linear models in R: the package dlnm. J Stat Softw. 2011:43:1-20.

27. Ecker MD, Gelfand AE. Bayesian variogram modeling for an isotropic spatial process. J Agric Biol Environ Stat. 1997:2:347-69.

28. Kuo L, Mallick B. Variable selection for regression models. Sankhyā Indian 」 Stat Ser B 1960-2002. 1998:60:65-81.

\section{Submit your next manuscript to BioMed Central and take full advantage of:}

- Convenient online submission

- Thorough peer review

- No space constraints or color figure charges

- Immediate publication on acceptance

- Inclusion in PubMed, CAS, Scopus and Google Scholar

- Research which is freely available for redistribution

Submit your manuscript at www.biomedcentral.com/submit 